Jan Priewe, Thomas H.W. Sauer

\title{
Grüne Wirtschaftspolitik ohne Reformprojekt ${ }^{1}$
}

\section{Der Strategiewechsel der Bündnisgrünen}

Die Bundestagswahlen 1998 brachten Bündnis 90/Die Grünen zwar nicht an die Macht, aber immerhin in die Regierung. Bereits wenige Monate nach dem Eintritt in die Bundesregierung begann sich jedoch ein Teil ihrer Wählerbasis enttäuscht von der grünen Partei abzuwenden. Die Gründe dafür sind wohl vor allem in der außen- und sicherheitspolitischen Kehrtwende zu suchen, welche die Bündnisgrünen mit ihrer mehrheitlichen Positionierung zum Kosovo-Krieg vollzogen haben. Die wirtschafts- und sozialpolitische Profilierung von Bündnis 90/Die Grünen spielt dagegen für das Wahlverhalten von jeher nur eine untergeordnete Rolle: Die Kompetenz, die den Grünen auf diesem Gebiet von der befragten Bevölkerung zugebilligt wird, ist traditionell eher gering. Die Grünen gelten als ,wirtschaftsfremde" Partei, sieht man von umweltökonomischen Fragestellungen ab. Dieses Manko wiegt schwer, da nahezu alle Politikbereiche immer stärker von ökonomischen Fragen durchdrungen werden.

Daher ist es nicht bedeutungslos, wie sich die Grünen auf wirtschafts- und sozialpolitischem Gebiet positionieren: Es macht durchaus einen Unterschied, ob sich der grüne Juniorpartner hier ,links“ oder „,rechts“ vom Seniorpartner SPD zu profilieren versucht - für die Erfolgsaussichten eines sozial-ökologischen Reformprojektes könnte das sogar von ausschlaggebender Bedeutung sein. Georg Bannas' insgeheimes Frohlocken in der FAZ über den Zustand der ,wachsweich“ gewordenen Grünen scheint zutreffend:

„Der Kanzler braucht nur mit der Stirn zu runzeln, und schon geben ihm die Wortführer der Grünen Recht. Schröder ist es gelungen, den Grünen seine Maßstäbe aufzunötigen .... Die Grünen wären nicht zum Regieren fähig geworden, hätten sie sich nicht angepaßt. Doch haben sie darüber ihre Meinungsführerschaft in öffentlichen Debatten verloren. Ihre Rolle ist nicht mehr innovativ, sondern verwaltend." (FAZ vom 19.8.1999)

1 Ein Teil dieses Aufsatzes ist die überarbeitete Fassung eines Textes von Jan Priewe, der in der Septemberausgabe der Zeitschrift Kommune unter dem Titel Rotgrüne Beschäftigungspolitik am Scheideweg: Arbeitslosigkeit bekämpfen oder Standort verwalten erscheint. 
Dabei gehört es zu den Paradoxien der gegenwärtigen politischen Situation, daß Bündnis 90/Die Grünen von der befragten Bevölkerung immer noch als Partei „links“ von der SPD wahrgenommen wird - zugleich aber maßgebliche Kreise innerhalb der grünen Partei im Verein mit Stimmen der veröffentlichten Meinung alles daran setzen, ihr die Erbschaft einer vermeintlich dahin siechenden FDP aufdrängen, frei nach dem Motto: An den Freiburger Thesen (der FDP) sollen die Grünen genesen. Es heißt: Die Grünen könnten nicht zugleich die Partei der Hausbesitzer und der Hausbesetzer sein. Dieser anvisierte Strategiewechsel läßt sich natürlich nicht ohne einen ,teilweisen Austausch der Mitgliedschaft", und des Wahlvolkes vollziehen. Es war daher auch nur konsequent, wenn dies jüngst stellvertretend von einigen Junggrünen gefordert wurde. Dabei wird bewußt in Kauf genommen, daß die PDS eine dann auf der Linken entstehende Vakanz dauerhaft füllen kann.

Abgesehen davon, daß sich Mitgliedschaft und Wahlvolk nicht nach Belieben austauschen lassen, wird dieser Strategiewechsel dadurch erschwert, daß bereits ein harter Wettbewerb darum läuft, wer denn die schönste Pose in der ,neuen“ Mitte einnimmt. Dieser Wettbewerb ist wenig originell, wie ein Blick über den bundesdeutschen Tellerrand zeigt. So schreibt Robert Reich in seinem Tagebuch über seine Zeit als Arbeitsminister im ersten Kabinett Clinton:

„Jeder Politiker in Amerika will, daß man von ihm sagt, er stehe in der Mitte. Wer will schon am Rande stehen? Etiketten wie ,rechts' oder ,links' gefährden die politische Karriere. Die Öffentlichkeit fühlt sich besser bei Leuten aufgehoben, die sich zur totalen Mäßigung bekennen. Besonders gilt das für Präsidenten. (...) Aber das sind bloß Posen“ (Reich 1999: 292).

Das Problem beginnt dort, wo die Pose mit realer Politik verwechselt wird, wie von denjenigen grünen und sozialdemokratischen Kreisen der Bundesrepublik, die sich um die Anerkennung durch die „neue“ Mitte balgen. Wird diese Verwechslung nicht erkannt, dann wird nichts anderes als konventionelle, letztlich die soziale Struktur konservierende Politik betrieben, wie Robert Reich für die USA deutlich macht:

„Praktisch jeder Versuch zu führen - Energie und Engagement der Amerikaner zu aktivieren - wird, wenn der Appell erstmals ergeht, immer unkonventionell sein. (...) Führen kann man per definitionem nicht von der Mitte aus, denn dann bedürfte es keiner Führung. Das Volk wäre schon da“" (Reich 1999: 292-293).

Dies gilt auch für Meinungsführerschaften. Die Pose der ,neuen“ Mitte hat im „Modernisierungs“-Diskurs ihr Manna gefunden. Das gilt in Deutschland vor allem für das Bild, das Schröder von den Perspektiven der Sozialdemokratie entwirft, aber auch für die eifrigen „Modernisierer“ innerhalb der grünen Partei. Doch dabei meint „Modernisierung“ immer nur

„einen Prozeß, der ohnehin abläuft und in den man sich nur einklinken muss. (...) Es gibt, so die Botschaft, eigentlich nichts zu erwägen und zu entscheiden, man muß bloß ausführen, was an der Zeit ist" (Jan Ross in der ZEIT vom 15.7.99: 3). 
Diese Vision einer entpolitisierten Welt wurde bereits von Roman Herzog in seiner oft zitierten „Berliner Rede“ mit der Formulierung auf den Punkt gebracht, es gäbe kein Erkenntnis- sondern lediglich ein Umsetzungsproblem. Politik hat aber sehr wohl viel mit konkurrierenden Analysen gesellschaftlicher Problemlagen und dem Abwägen von alternativen Lösungsansätzen, mit Entscheidungen und der Mobilisierung von gesellschaftlichen Mehrheiten für die Durchsetzung dieser Entscheidungen zu tun. Wer dies übersieht, wer die Pose der ,neuen“ Mitte bereits für Politik und „Modernisierung" für einen Wert an sich hält, der wird im Ergebnis konservative Politik betreiben, nur eben unter anderem Etikett:

„Der Grundfehler der Modernisierungsdoktrin ist, dass sie .. mit dem Neuen nur scheinbar auf guten Fuß steht, während sie in Wahrheit bloß die Linien aus der Vergangenheit in die $\mathrm{Zu}$ kunft ausziehen will. Ihr Leitmotiv ist nicht Neugier, Wirklichkeitslust, Fantasie, sondern letztlich das Weiter-so“ (Jan Ross in der ZEIT vom 15.7.99: 3).

Neugier, Wirklichkeitslust und Fantasie waren Eigenschaften mit denen die Grünen einstmals identifiziert wurden: die Neugier auf neue soziale Entwicklungen und Bewegungen, die Lust, die Wirklichkeit im Lokalen und Dezentralen auch jenseits des Marktgeschehens wirklich zu verändern, die Fantasie, durch konkrete basisdemokraktische und gewaltfreie Ansätze „von unten herauf“ auch die globale soziale und ökologische Realität zum Besseren zu wenden, die Fähigkeit, Zukunftsfragen zu thematisieren.

Neugier, Wirklichkeitslust und Fantasie wären auch notwendige Ingredienzen eines sozial-ökologischen Reformprojekts: Neugier, die Träger und Triebkräfte globaler wirtschaftlicher, sozialer und ökologischer Entwicklung tatsächlich zu verstehen, die Lust, alternative Entwicklungspfade zu formulieren und durchzusetzen, sowie die Fantasie, die sozialen Subjekte dieser überfälligen Veränderungen anzusprechen und zu mobilisieren, mit ihnen gemeinsam den öffentlichen Raum für die Verständigung über die sozialen und ökologischen Ziele der Reformen zu erobern sowie die geeigneten Mittel für ihre Umsetzung zu finden und auch anzuwenden.

Aber mit der Neugier, Wirklichkeitslust und Fantasie der Grünen verhält es sich wie mit der Riesengestalt Tuturs in einem Kinderbuch von Michael Ende: Je näher sich Jim Knopf und Lukas, der Lokomotivführer, ihm annähern, um so mehr schrumpft er auf normales menschliches Maß zusammen, bis sich herausstellt, daß es sich lediglich um einen armen, einsamen Scheinriesen handelt. Je näher die Grünen an die vermeintliche Macht auf Bundesebene kamen, um so mehr schrumpften die Eigenschaften, die sie eigentlich als Motoren eines sozial-ökologischen Reformprojekts prädestinierten, auf ein langweiliges Normalmaß: Die Neugier auf die verändernde Kraft neuer sozialer Bewegungen wurde zu einer artigen Beflissenheit gegenüber dem guten, alten deutschen „Mittelstand“; die Lust, Wirklichkeit $\mathrm{zu}$ verstehen und zu verändern, wurde durch ein Nachbeten oberflächlicher 
Analysen wirtschaftlicher Globalisierung ersetzt, das aus Ansätzen alternativer Politik eine Politik vermeintlicher Alternativlosigkeit macht; schließlich ist die Fantasie, den Individuen Freiräume für ihre Vergemeinschaftung jenseits von Markt und Staat zu erobern, einer Art Gottvertrauen auf die Selbstregulierungsfähigkeit des Marktes gewichen.

Wie konnte es zu diesem spezifisch grünen "Scheinriesen“-Syndrom“ kommen? Die einfachste Erklärung wäre: „Macht korrumpiert“. Aber diese Erklärung ist zu einfach. Schließlich wird jede Partei, je näher sie der Regierungsverantwortung rückt, ihre Positionen auf Widerspruchsfreiheit, Praktikabilität und Mehrheitsfähigkeit überprüfen müssen. Außerdem werden die Mandate, die diese Partei zu vergeben hat, um so interessanter, je wahrscheinlicher die Übernahme von Regierungsverantwortung wird. Es ist einer kleinen Partei auch nicht vorzuwerfen, daß sie engagierten und kompetenten Quereinsteigern bessere Aufstiegschancen eröffnet als die großen Volksparteien. Problematisch wird es für die grüne Partei erst dann, wenn sich ihre strategischen Entscheidungszentren in informellen Zirkeln verselbständigen, die durch keinerlei demokratische Strukturen mehr legitimiert sind und mit den formalen Parteigremien nur noch durch eine dünne ideologische Decke verbunden sind.

Wesentlicher für den sich bei den Grünen vollziehenden Strategie- und Programmwechsel erscheint uns aber ein anderer Grund: Die mit Schrecken wahrgenommene Auflösung des vor allem großstädtisch geprägten alternativen Milieus, das die Öko-Partei in den achtziger Jahren in die Parlamente trug, veranlaßte einflußreiche grüne Strategen zur Suche nach einem anderen Wählerpotential. Dabei wird die innovative Melange des einstmals alternativen Milieus von ökologischen Fundamentalisten, linken Intellektuellen, feministischen, pazifistischen, öko-libertären und wertkonservativen Strömungen immer weniger als wertvoller Ausgangspunkt für eine unkonventionelle Verbreiterung der Wählerbasis empfunden, sondern als Risiko bei der Übernahme des ,mittelständischen“ Wählerpotentials der FDP. Die Chance, eine von der Dominanz einzelner Klientel freie, sozial-ökologische Politik zu entwickeln, die zum Anziehungspunkt von undogmatischen, innovativen Akteuren aus neuen und alten sozialen Bewegungen, aus Gewerkschaften, Wirtschaftsverbänden, Wissenschaft und Kultur werden könnte, wird so verspielt. Statt dessen führt die anvisierte strategische Neuausrichtung der grünen Partei auf die „Wechselwähler“ der imaginären neuen Mitte zu einer Politik, die sich immer stärker an den Eliten in Wirtschaft, Medien und Wissenschaft orientiert.

Ein weiterer Blick in die USA verdeutlicht diese Entwicklung. Dort zeichnete sich bereits unter der ersten Regierung Clinton ein Trend im Wählerverhalten $a b$, mit dem sich ein solcher Strategiewechsel rechtfertigen läßt: Die Reichen in den USA gehen immer häufiger zur Wahl, während sich die 
Menschen in der unteren Hälfte der Einkommensskala immer seltener an den Wahlen beteiligen. Das geschieht vor dem Hintergrund einer immer größer werdenden Kluft zwischen hohen und niedrigen Einkommen. Bei insgesamt stagnierenden (oder inzwischen vielleicht geringfügig steigenden) Durchschnittseinkommen finden die Beschäftigten in der unteren Einkommenshälfte immer weniger in der Lohntüte. Am Boom der Aktienmärkte dagegen haben die wohlhabendsten zehn Prozent der Bevölkerung einen Anteil von siebzig Prozent. Robert Reich kommentiert diese Entwicklung folgendermaßen:

„Nimmt man all das zusammen, dann kommt der größte Teil des Wirtschaftswachstums weiterhin den Leuten ganz oben zugute. Die untere Hälfte verliert laufend an Boden. Und sie geht immer seltener wählen. Ein Teufelskreis. Die Politik belohnt oft die Reichen, weil sie sich wirksamer an ihr beteiligen, und dies wiederum veranlaßt sie zu noch mehr Beteiligung. Die Politik bestraft oft die untere Hälfte, weil sie sich immer weniger an ihr beteiligt und ... das Ergebnis bestätigt dann ihren Zynismus.“ (Reich 1999: 379)

Diese Entwicklung in den USA läßt erahnen, was auch auf die Bundesrepublik zukommt, wenn die deutschen Grünen im Verein mit der Sozialdemokratie an ihrem Kurswechsel auf den vermeintlich sicheren Hafen der „,neuen“ Mitte festhalten: Ihre Sozial- und Beschäftigungspolitik wird endgültig von der Perspektive der Betroffenen zu einer Perspektive der Verwaltung von Arbeitslosigkeit und sozialer Ungleichheit wechseln. Die einstmals auf Emanzipation und Partizipation ausgerichteten Ansätze der Grünen können dieses elitäre Projekt nur noch stören.

Die Vorstellungen der Strategen der ,neuen“ Mitte, nach denen Parteien als „politische Dienstleistungsunternehmen“ definiert und das Primat der Senkung der Staatsquote gegenüber allen anderen politischen Zielen durchgesetzt werden sollen, sind mit einem sozial-ökologischen Reformprojekt jedenfalls nicht zu vereinbaren. Statt dessen versuchen diese Strategen, ein ganz normales Sparpaket als ,größtes Reformprojekt aller Zeiten“ (Originalton Schröder) zu verkaufen.

Die grünen Haushaltspolitiker stellt der Wechsel von Lafontaine zu Eichel allerdings vor ein Problem: Sie können sich gegenüber dem neuen Bundesfinanzminister, der sich schnell als ,ehrlicher Makler der Haushaltskonsolidierung" einen Namen machte, und Schröder, der mit der Rolle des „Austeritäts-Kanzlers“ keine Probleme hat, kaum noch als die konsequenteren Vertreter einer ,nachhaltigen Finanzpolitik“" profilieren, und das, obwohl sie doch diesen netten, ideologielastigen Begriff geprägt hatten. Wie wenig originell der sich anbahnende rot-grüne Wettbewerb um den „Preis des besseren Defizit-Falkens" ist, zeigt wiederum ein Blick über den NordAtlantik. Robert Reich (1999: 134) weist darauf hin, daß alle erstmals gewählten demokratischen Präsidenten der USA ,Defizit-Falken“ waren, in dem Sinne, daß sie nach der Amtsübernahme die Haushaltskonsolidierung 
zu ihrer obersten Priorität machten: Hatte Clinton im Wahlkampf noch Milliarden US-Dollar öffentlicher Investitionen in die Bereiche Bildung und Gesundheit versprochen, blieb nach seiner Mutation zum „Defizit-Falken“ wenig von den versprochenen Programmen übrig: Der Clinton-Haushalt verkündete ganz Amerika vielmehr,

„der Ausweg aus allen wirtschaftlichen Problemen liege in der Reduzierung des Defizits und einer geringeren Schuldenaufnahme der öffentlichen Hand - egal, für welchen Zweck das Geld bestimmt ist. Diese Logik kennt keine Grenzen mehr, ihr ist nicht mehr zu entkommen. Das konzeptionelle Gefängnis ist komplett.“ (Reich 1999: 174)

Die grünen Haushaltspolitiker haben das ihre dazu beigetragen, auch in der Bundesrepublik ein derartiges „konzeptionelles Gefängnis“ zu errichten. Finanzminister Eichel hat es jetzt endgültig geschlossen: Gefangen darin ist eine sozial-ökologisches Reformprojekt, das der Verminderung der Arbeitslosigkeit in Deutschland oberste Priorität einräumt.

\section{Arbeitslosigkeit und ,grüne Austeritätspolitik“}

Seit über 20 Jahren ist Massenarbeitslosigkeit nicht nur in Deutschland sondern in der ganzen Europäischen Union das zentrale soziale Problem und so wird es wahrscheinlich auch noch über Jahre hinweg bleiben. Zwar ist Arbeit beileibe nicht alles, doch ohne ausreichende Möglichkeiten zur Erwerbsarbeit wird die Gleichstellung der Geschlechter nicht vorankommen, werden die sozialen Sicherungssysteme überfordert, die Staatsfinanzen unterhöhlt und die Chancen für einen sozialverträglichen ökologischen Umbau vermindert. Auch die ökonomische Spaltung zwischen West- und Ostdeutschland wird sich nicht grundlegend ändern, solange es eine im Osten viel höhere Arbeitslosigkeit als im Westen gibt. Eine wesentliche Verminderung der Arbeitslosigkeit ist somit die zentrale Voraussetzung für jedes ernsthafte sozial-ökologische Reformprojekt.

Das Problem der Arbeitslosigkeit wird bei den Grünen inzwischen aber weitgehend durch eine neoliberale Brille wahrgenommen. Betrachtet man die konzeptionellen Grundlagen der von den Grünen geforderten ,nachhaltigen" Haushalts- und Finanzpolitik, so findet sich mittlerweile nur noch die kritiklose Übernahme neoklassisch inspirierter Ideen:

„Der Abbau der Arbeitslosigkeit kann nur erreicht werden, wenn auf der Grundlage einer nachhaltig ausgerichteten wirtschaftlichen Entwicklung mit Strukturreformen auf den Güterund Faktormärkten bestehende Inflexibilitäten beseitigt werden“ (Bündnis 90/Die Grünen im Bundestag, 8.3.1999, S. 12).

Die gängige neoklassische Analyse des Arbeitsmarktes sieht den wesentlichen Grund der ,unfreiwilligen Arbeitslosigkeit“ schlicht und einfach in einem zu hohen Reallohnniveau (und nicht etwa in einer fehlenden Nachfrage, diese sei nur von untergeordneter Bedeutung). Daß das Reallohnniveau auf einer Höhe bleibt, die eine Markträumung (Vollbeschäftigung) 
verhindert, wird als Ausdruck eines gestörten Wettbewerbs aufgefaßt: Der Arbeitsmarkt ist zu wenig flexibel als daß sich ein Reallohnniveau herausbilden kann, bei dem Vollbeschäftigung herrscht. Diese Inflexibilität sei institutionellen Bedingungen geschuldet, die ein Sinken der Reallöhne verhindern: zu hohe Lohnersatzleistungen, zu hohe Lohnnebenkosten (zu viel „Sozialstaat“), Flächentarifverträge, zu starke und monopolistisch agierende Gewerkschaften („Lohnkartell“), zu viel Kündigungsschutz, zu starres Recht etc.. Insofern sei die Arbeitslosigkeit im wesentlichen „strukturell““ bedingt.

Bei einer solchen Argumentation erscheint Arbeitslosigkeit als ein Phänomen, das im wesentlichen in den mikroökonomischen Bedingungen des Arbeitsmarktes begründet ist und auch nur dort beseitigt werden kann. Bei sinkenden Reallöhnen könnte Arbeitslosigkeit demnach auch ohne höheres Wirtschaftswachstum beseitigt werden, da in der Produktion einfach mehr Arbeit eingesetzt würde. Stimmt die Mikroökonomie, so die implizite These, dann braucht man sich um die makroökonomischen Bedingungen nicht zu kümmern.

Die „Arbeitsgruppe Haushalt“ der grünen Bundestagsfraktion überträgt diese Analyse auch auf die europäische Ebene. Auch hier sei das „Problem der hohen Arbeitslosigkeit überwiegend strukturell bedingt" (ebd.: 13), wie sie zustimmend die Europäische Zentralbank (EZB) zitiert. Die EZB könne mit ihrer Zinspolitik daher auch keine unmittelbare Verantwortung für die Arbeitslosigkeit übernehmen, diese sei vielmehr an die nationalen Regierungen zurückzuverweisen. „Ein idealer Policy-Mix für die Euro-Zone“ bestünde - nach Meinung der grünen Haushaltspolitiker - aus folgender Kombination: „Konsequente Strukturreformen in den Mitgliedsländern, verbunden mit einer Politik der finanzpolitischen Konsolidierung“" (ebd.). Im Gegenzug dazu würden sich die Voraussetzungen für eine weniger strikte Geldpolitik der Europäischen Zentralbank quasi automatisch ergeben. Ein Lafontaine, der öffentlich nach Zinssenkungen ruft, wird aus dieser Sicht natürlich überflüssig.

Bemerkenswert ist an dieser Wendung, daß die grünen Haushaltspolitiker die Verantwortung für die Beschäftigungspolitik an die europäischen Nationen zurückverweisen, nachdem die Grünen anderthalb Jahre zuvor der Einführung des Euros nur unter der Prämisse zugestimmt hatten, daß die Währungsunion durch eine Beschäftigungs- und Sozialunion ergänzt wird. Davon ist jetzt keine Rede mehr. Statt dessen soll der Europäische Stabilitäts- und Wachstumspakt jetzt zur verbindlichen ,Leitlinie für eine nachhaltige Finanzpolitik" gemacht werden.

Dem ist entgegenzuhalten, daß eine beschäftigungspolitische Konzeption, die gesamtwirtschaftlich durchdekliniert wird, in den entscheidenden geld-, fiskal- und lohnpolitischen Teilen nur auf der supranationaler Ebene der 
Euro-Länder realisiert werden kann. Der „Europäische Beschäftigungspakt" bietet dafür jedoch kaum ernsthafte Ansatzpunkte. Es sei an dieser Stelle nur auf die Währungspolitik hingewiesen: Die weltwirtschaftlichen Turbulenzen der letzten zehn Jahre haben ganz wesentlich mit einer gefährlichen Architektur der Weltfinanzen zu tun, deren Risiken zwar in erster Linie auf die Schwellen- und Transformationsländer bzw. die Schwachwährungsländer abgewälzt werden, doch schlagen die Probleme auf stark außenhandelsabhängige Länder wie Deutschland ganz unvermeidlich zurück. Zwar reden auch die Grünen gerne von Globalisierung, doch gedacht und gehandelt wird nach wie vor provinziell, eine europäische Initiative bleibt außen vor.

Die Prioritäten der grünen Haushaltspolitiker liegen an anderer Stelle. Sie halten es für „erforderlich, daß der vorgesehene Kurs der Ausgaben- und Defizitbegrenzung auf allen Haushaltsebenen auch beim Auftreten unerwarteter neuer Haushaltsbelastungen eingehalten wird“ (ebd.: 11). Dazu werde auch auf nationaler Ebene ein ,wirksamer Stabilitätspakt benötigt“ (ebd.). Die Konsequenz der geforderten Senkung der Defizitquote auf Null ist, daß bei gewünschter Konstanz der Abgabenquote die Staatsausgaben langsamer als das Sozialprodukt wachsen bzw. bei gewünschter Senkung der Steuer- und/oder Abgabenquote die Staatsausgaben besonders stark gedrosselt werden. Wo gespart werden soll und wie dies zu steigender Beschäftigung führen soll, wird allerdings nicht erklärt.

Das von den grünen Haushaltspolitikern übernommene Konzept beruht auf einer Reihe stillschweigender Voraussetzungen, die von erheblicher politischer Brisanz sind. So wird ein Trend sinkenden bzw. anhaltend niedrigen Wachstums hingenommen - nicht etwa, weil Wachstum für unvereinbar mit ökologischer Nachhaltigkeit gehalten wird, sondern weil es staatlicherseits offenbar nicht beeinflußt werden kann oder soll. Das ist eine klassische laisser-faire-Position. Bei nachlassendem Wachstum ist ein konstantes staatliches Leistungsniveau an öffentlichen Gütern und Transfers aber nur mit steigender Staatsquote oder steigender Verschuldung finanzierbar. Wird beides abgelehnt, dann müssen staatliche Leistungen zurückgefahren werden, und es ist äußerst blauäugig zu meinen, dieser Sparkurs wäre ohne Abbau sozialstaatlicher und ökologisch relevanter Ausgaben zu bewerkstelligen.

Hier wird aber nun weiter unterstellt, daß das angeblich unumgängliche staatliche Sparen keine negativen gesamtwirtschaftlichen Auswirkungen hat - entweder weil die geringeren Staatsausgaben preisdämpfend wirken und somit das reale Sozialprodukt nicht vermindern würden, oder weil die staatliche Sparpolitik zu sinkenden Zinsen führen würde. Letzteres ist jedoch äußerst unsicher und ersteres würde bei der gegenwärtig erreichten Preisstabilität zu einer deflationären Entwicklung führen. Deflation erhöht 
aber die Realzinsen, steigert somit die Schuldenlast der Privaten und des Staates und verschlechtert die für die Investitionstätigkeit maßgeblichen langfristigen Erwartungen der Unternehmen. Restriktive Wirkungen starker staatlicher Ausgabenkürzungen einfach abzustreiten, erscheint reichlich naiv. Kommt es jedoch zu restriktiven Wirkungen auf Produktion und Beschäftigung, dann werden auch die erhofften Konsolidierungseffekte nicht eintreten.

Während die restriktiven Auswirkungen der Sparpolitik bagatellisiert werden, gilt umgekehrt eine expansive Fiskalpolitik als wirkungslos oder gar schädlich. Als Begründung reicht der stereotype Hinweis auf das Scheitern keynesianischer „Globalsteuerung“ via kreditfinanzierter Ausgabenprogramme in den 70er Jahren. Was an diesen Programmen tatsächlich ,keynesianisch" war und was moderne makroökonomische Konzeptionen anzubieten haben, die an europaweit koordinierten, kreditfinanzierten Investitionsprogrammen und einer Veränderung der Geldpolitik ansetzen, das braucht dann alles nicht mehr diskutiert zu werden.

Die sozusagen empirische Plausibilität gewinnen die nun auch von den Grünen aufgenommenen Sparappelle durch den enorm gestiegenen Schuldendienst im Bundeshaushalt. Am meisten zur Staatsverschuldung beigetragen haben jedoch die Kosten der deutschen Einigung sowie die im Anschluß daran von der Zinspolitik der Bundesbank ausgelöste Rezession. Die Zinslast schränkt den Spielraum für normale Staatsaufgaben in der Tat stark ein, und in der Vergangenheit haben die Bündnisgrünen zu Recht die einseitige Finanzierung der deutschen Einigung über Kredite und die Erhöhung der Lohnnebenkosten kritisiert. Allerdings stellt sich die Frage, wie auf diese außergewöhnlichen Kosten, die auch noch lange anhalten werden, reagiert wird: durch eine Sparpolitik um jeden Preis oder durch Steuermehreinnahmen. Nachdem seit den 80er Jahren die oberen Einkommensklassen eine ganz erhebliche Steuer- und Abgabenentlastung erfahren haben, wäre eine Sondersteuer zur Bewältigung dieser Kosten, etwa eine Vermögensabgabe ergänzend zum Solidarzuschlag, bei der Einkommenssteuer durchaus angebracht. Aber offenbar haben es die Bündnisgrünen längst aufgegeben, über eine Alternative zur Finanzierung der deutschen Einigung, wie sie von der konservativ-liberalen Regierung konzipiert wurde, auch nur nachzudenken.

Inzwischen ist es auch Mode geworden, grüne Austeritätspolitik mit dem Verweis auf intergenerative Gerechtigkeit zu begründen: Staatsverschuldung würde zukünftige Generationen be- und die jetzigen entlasten. Tatsächlich stehen jedoch den Zahlern in jeder Periode auch Empfänger in der gleichen Periode gegenüber, so daß es sich (da der Anteil der Auslandsschulden gering ist) zunächst einmal um intragenerative Umverteilungsvorgänge handelt. Um diese korrekt zu beurteilen, müßten die Vertei- 
lungswirkungen einer Ausgabenkürzung oder umgekehrt einer Steuererhöhung verglichen werden. Schließlich sei vor der Beschwörung simpler Generationskonflikte gewarnt. Die Last der Arbeitslosigkeit trägt die jetzige Generation, insbesondere in Ostdeutschland. Die Einigungslasten sollten aber wohl nicht allein von der jetzigen Generation getragen werden, handelt es sich doch um ein Jahrhundertereignis. Von den zu erwartenden Erbschaften infolge der Vermögensbildung der letzten Jahrzehnte wollen wir gar nicht erst reden. Kurzum: Das Argument der intergenerativen Gerechtigkeit ist nicht mehr als das legitimatorische Feigenblatt für eine grüne Austeritätspolitik.

\section{Fortsetzung neoliberaler Politik}

Im jüngsten Dokument grüner Wirtschaftspolitik, der im März 1999 vorgelegten Initiative für Investitionen, Arbeit und Umwelt (Berninger u.a. 1999), werden die Konturen künftiger Bestrebungen deutlich: Neben einer scharfen Haushaltskonsolidierung, die über Einsparungen in allen Bereichen erreicht werden soll (Steuererhöhungen sollen auf jeden Fall vermieden werden), Innovationen und ökologischem Strukturwandel, wird zum Abbau der Arbeitslosigkeit vor allem eine Reform der Unternehmensbesteuerung, gewerkschaftliche Lohnzurückhaltung sowie eine Senkung der Lohnnebenkosten gefordert - Kernstücke der bereits von der KohlRegierung vertretenen neoliberal orientierten Politik. In der veröffentlichten Meinung gelten zwar Unternehmenssteuern und Lohnnebenkosten als die Hemmnisse schlechthin für mehr Beschäftigung; doch handelt es sich dabei um fundamentale Irrtümer mit fatalen Folgen.

Für eine Reform der Unternehmenssteuern sprechen zwar viele gute Gründe, ob eine solche Reform aber zu mehr Investitionen und mehr Beschäftigung führt, ist äußerst zweifelhaft. So sinkt in Deutschland seit über zwei Jahrzehnten im Trend die Dynamik der privaten Investitionen - obwohl es gerade in diesem Zeitraum zu einer massiven steuerlichen Entlastung gekommen ist. Vor kurzem erklärte der Bundeswirtschaftsminister sogar, daß die Steuerzahlungen des Unternehmenssektors geringer seien als die empfangenen Subventionen. Wer glaubt, durch Steuerentlastungen den Unternehmen zu mehr Gewinnen verhelfen zu müssen, unterstellt, daß hier die Restriktionen für die Investitionen liegen. Dagegen steht jedoch, daß die Renditen, egal wie sie berechnet werden, ebenso wie der Selbstfinanzierungsgrad der Investitionen im Trend seit langem ansteigen. Das Problem sind nicht zu geringe Gewinne der Unternehmen, sondern die Art und Weise ihrer Verwendung.

Wird trotzdem eine Unternehmenssteuerreform durchgeführt, die den Unternehmenssektor entlastet, so vermindern sich die Staatseinnahmen und es 
müßte entweder die Staatsverschuldung erhöht werden, was die Bündnisgrünen ablehnen, oder es müßte an anderer Stelle eingespart bzw. irgendeine andere Steuer erhöht werden. Während man bei einer Kreditfinanzierung der Steuerentlastung annehmen kann, daß die Beschäftigung zumindest kurzfristig anstiege, bleibt der Nettoeffekt bei einer Umschichtungsfinanzierung vollkommen unsicher: Er kann durchaus auch negativ sein! Von den Gewerkschaften wird „Lohnzurückhaltung“ gefordert, worunter üblicherweise verstanden wird, daß die Reallöhne mit einer geringeren Rate wachsen sollen als die Produktivität. Damit wird die Hoffnung verbunden, daß ,Investitionen im Standort Bundesrepublik kalkulierbar und damit attraktiver" (ebd.: 11) würden. Vergessen bleibt, daß wir eine solche „Lohnzurückhaltung“ im Trend seit 1982 in Westdeutschland bereits haben, ohne daß dies zu einer Verminderung der Arbeitslosigkeit geführt hätte. Hier helfen, wie die Vergangenheit zeigte, auch keine Absprachen mit Arbeitgeberverbänden. Im Normalfall führen relativ zur Produktivität sinkende Löhne zu geringerer Inflation bzw. bei bereits erreichter Preisniveaustabilität wie in Europa zu Deflation.

Als „success story“ für die arbeitsplatzschaffende Wirkung gewerkschaftlicher Lohnzurückhaltung müssen immer wieder die Niederlande herhalten. Allerdings führte die Lohnzurückhaltung dort nur deshalb nicht zur Deflation, weil die Exportüberschüsse zu Lasten der Nachbarländer enorm angestiegen sind. Das heißt, es handelte sich hier um eine klassische „,beggarmy-neighbour"-Politik, die ein kleines Land ohne allzu schlimme Folgen betreiben mag (da es sich seine großen Nachbarn leisten können, diese Politik zu tolerieren); für Deutschland und Europa wäre sie jedoch fatal: Eine von Deutschland ausgehende Spirale von Lohnsenkung und Deflation müßten die übrigen EU-Länder nachvollziehen, so daß es erst recht zu Stagnation und Arbeitslosigkeit käme. Was die grünen Vertreter hier formulieren, ist in der Konsequenz leichtsinnig und gefährlich. Dadurch wird all das, was gleichzeitig über die Notwendigkeit weiterer Arbeitszeitverkürzungen in den verschiedensten Formen gesagt wird, gänzlich entwertet. Schließlich wird auf die Senkung der Lohnnebenkosten durch Lohnkostensubventionen für Niedrigeinkommen und Ökosteuern gesetzt. Zwar signalisiert die Höhe der Lohnnebenkosten einen Reformbedarf bei der Finanzierung sozialpolitischer Leistungen, doch folgt aus einer Reduzierung der Lohnnebenkosten keineswegs eine steigende Beschäftigung, denn genauso wie bei einer allgemeinen ,Lohnzurückhaltung“ ist bei einer Senkung der Lohnnebenkosten zunächst einmal von einem Deflationseffekt auszugehen, der sich besonders stark auswirkt, wenn diese Senkung durch eine Verminderung der Sozialversicherungsleistungen wie der Renten finanziert wird. Sollen die Lohnnebenkosten aber nicht durch Leistungskürzung sondern durch Lohnsubventionen im Niedriglohnbereich vermindert werden, dann 
müßte den - im günstigsten Fall - entstehenden neuen Arbeitsplätzen allerdings die wegfallende Beschäftigung gegenübergestellt werden, denn um diese Subventionen zu finanzieren muß bei anderen Staatsausgaben gespart werden. Werden die Lohnnebenkosten über eine Subventionierung des Sozialversicherungssystem durch eine Ökosteuer (also eine Kostenerhöhung an anderer Stelle) gesenkt, dann kommt es zu komplizierten Wirkungsketten, bei denen der Beschäftigungseffekt nur schwer zu kalkulieren ist. Sowohl die Beschäftigungs- als auch die Umwelteffekte einer Ökosteuer wurden von grüner Seite in der Vergangenheit maßlos überschätzt (vgl. dazu Priewe 1998).

Es bleibt schließlich die Diskussion über den Zusammenhang von Umweltschutz und Beschäftigung. Tatsächlich kann diese Rechnung unter bestimmten Bedingungen aufgehen, dabei geht es aber um kleine Quantitäten. Etwa drei Prozent der gegenwärtigen Beschäftigung in Deutschland werden dem Umweltschutz zugerechnet. Die Tendenz ist schon wieder rückläufig. Mehr als ein Plus von 500.000 Arbeitsplätzen ist selbst unter optimistischsten Bedingungen in ein paar Jahren kaum zu erwarten, und selbst dabei ist nicht ganz sicher, ob es um Brutto- oder Nettozahlen geht.

Umweltschutz, gleich durch welche Instrumente initiiert, führt nur dann zu mehr Beschäftigung, wenn entweder eine zusätzliche gesamtwirtschaftliche Nettonachfrage nach Gütern und Dienstleistungen entsteht, so daß es zu einem Anstieg des Sozialproduktes kommt, oder wenn durch Umschichtungen in den Ausgaben von Unternehmen, öffentlichen und privaten Haushalten eine Verminderung der gesamtwirtschaftlichen Arbeitsproduktivität je Stunde und damit eine Steigerung der durchschnittlichen Arbeitsintensität der Produktion eintritt. Beides kann, muß aber nicht der Fall sein. Umweltschutz kann auch beschäftigungsmindernd wirken - und gleichwohl notwendig sein. Die negativen Beschäftigungsfolgen müssen dann mit anderen Maßnahmen aufgefangen werden.

Alles spricht dafür, Umweltschutz und Beschäftigung mit jeweils separaten Instrumenten anzustreben. Es war daher ein folgenschwerer Fehler, auf die Ökosteuer als Zentrum eines sozialökologischen Reformprojektes zu setzen, und zu erwarten, man könne zwei Fliegen mit einer Klappe schlagen. Unter dem Strich kommt dabei ökologisch wie beschäftigungspolitisch nur wenig mehr als nichts heraus.

\section{Makroökonomische Rahmenbedingungen eines sozial-ökologischen Reformprojektes}

Nachdem sich in den 16 Jahren der konservativ-liberal geführten Regierung zeigte, daß die neoklassisch inspirierten Rezepte weder zum Abbau der Arbeitslosigkeit noch zu einer auch nur ansatzweisen Bewältigung der 
ökologischen Probleme geführt haben, wäre es an der Zeit, diese Rezepte grundsätzlich zu überdenken, statt sie einfach nur mit ökologischer Einkleidung fortzuschreiben, wie dies derzeit bei den Grünen geschieht. Dabei wäre es zentral, sich von der im Grunde nur mikroökonomischen Betrachtung der Mainstream-Neoklassik zu lösen und wieder stärker makroökonomische Zusammenhänge zu berücksichtigen. Dazu einige Stichpunkte.

Mittelfristig wird mehr Beschäftigung ohne ein stärkeres Wirtschaftswachstum nicht zu erreichen sein. So notwendig der Kampf für Arbeitszeitverkürzungen auch ist, die Arbeitszeitpolitik wird überlastet, wenn sie allein das Beschäftigungsproblem lösen soll. Die beiden vergangenen Jahrzehnte haben gezeigt, daß Arbeitszeitverkürzungen von mehr als 1\% pro Jahr kaum durchsetzbar sind. Sofern sie kostenneutral sein sollen, wären starke Arbeitszeitverkürzungen nur mit entsprechenden Einkommenseinbußen machbar, und dies vor allem bei unteren und mittleren Einkommensschichten. Wesentlich mehr Beschäftigung wird es bei einer nach wie vor starken Zunahme der Produktivität mittelfristig ohne Wachstum nicht geben. Durch ökologischen Strukturwandel, also Umschichtungen der Ausgaben von Haushalten und Unternehmen, sind wahrscheinlich nur geringe Beschäftigungszuwächse zu erreichen.

Auch die (wachstumsunabhängige) Schaffung eines Sektors niedriger Produktivität und niedriger Löhne ist wohl kaum ein gangbarer Weg. Zum einen könnte dieser Sektor wohl nur über einen radikalen, aber sozialpolitisch völlig unakzeptablen Abbau von Lohnersatzleistungen geschaffen werden - die Betroffenen wären dann wie in den USA zu nicht existenzsichernder „Armutsarbeit“ gezwungen. Zum anderen wären diese Niedriglohnarbeitsplätze für schlecht Qualifizierte allein schon deshalb prekär, weil sie immer wieder der Rationalisierung zum Opfer fielen (vgl. zu diesem Thema Bender u.a. 1999).

$\mathrm{Da}$ Wachstum aus ökologischen Gründen abzulehnen sei, gehört zur grünen Weltanschauung der Gründerjahre. Dagegen ist jedoch einzuwenden, daß Wirtschaftswachstum durchaus mit ökologischen Nachhaltigkeitszielen vereinbar ist, insoweit letztere klar in Form ökologischer Leitplanken und Umweltqualitätsziele definiert werden (vgl. Priewe 1998b). Anspruchsvolle Umweltqualitätsziele, die in einem mittelfristigen Nationalen Umweltplan festzuschreiben wären, müssen zu steigender Ressourceneffizienz und zu einem starkem Strukturwandel in Richtung auf die Erzeugung von „Umweltgütern" und wenig naturverbrauchenden Produktions- und Dienstleistungszweigen führen. Nur wenn alle Verbräuche von "Stoffen“ (oder throughputs) gleich welcher Art radikal und pauschal reduziert werden müßten, so daß ressourceneffizienzsteigernde Substitutionsvorgänge zwischen den verschiedenen Ressourcen ausgeschlossen würden, käme man an einer systematischen Senkung des Sozialprodukts - Nullwachstum würde 
keineswegs ausreichen - nicht vorbei. Wichtiger als die pauschale Senkung des Verbrauchs von allen "Stoffen“ ist jedoch der langfristige Übergang zu einem Energiesystem, das nahezu ausschließlich auf erneuerbaren Quellen beruht. Fossile Energieträger sind die entscheidende Naturknappheit (abgesehen von Wasser und Boden). Die Umstellung der Energieversorgung auf erneuerbare Träger ist das zentrale technologische Projekt des 21. Jahrhunderts. Wenn die ökologischen Leitplanken aufgestellt sind und bei einem höherem Sozialprodukt zu Bruch zu gehen drohen, dann muß allerdings auch das Wachstum beschränkt werden.

Ist Wachstum jedoch ökologisch möglich und zur Beseitigung der Arbeitslosigkeit unumgänglich, dann stellt sich die Frage, wie es jenseits der gescheiterten „Angebotspolitik“ (im Sinne von Deregulierung, Privatisierung, Arbeitsmarktflexibilisierung etc.) gefördert werden kann. Freilich sind institutionelle Reformen der verschiedensten Art sowie Strukturpolitik notwendig: Anreize für sinnvolle Produktion und Beschäftigung müssen durch entsprechende Institutionen geschaffen werden, wo sie nicht existieren. Allerdings brauchen institutionelle Reformen Zeit, und ihre Wirkung auf Wachstum und Beschäftigung ist unsicher. Darüberhinaus fördert eine allgemeine Innovationsorientierung in der Regel Wachstum und Produktivität gleichermaßen, so daß der Beschäftigungsgewinn relativ gering ist.

Die entscheidenden Ansatzpunkte zur Beeinflussung von Wachstum und Beschäftigung liegen - abgesehen von der Arbeitszeitverkürzung - vor allem bei der Geld-, Fiskal- und Lohnpolitik, deren Wirkung um so stärker ist, je größer die internationale Kooperation in diesen Feldern ist.

Die Geldpolitik hat kurz- wie langfristig erhebliche Auswirkungen auf Wachstum und Beschäftigung. Die entscheidende Rolle kommt dabei den Zinsen zu, die maßgeblich von der Europäischen Zentralbank beeinflußt werden. Die Kapitalmarktzinsen sind zwar nicht die einzige, aber eine wichtige Determinante der Investitionstätigkeit. Die Geldmenge, die von der Zentralbank bereitgestellt wird, muß für ein zu hoher Beschäftigung passendes Produktionspotential ausreichend sein, was nur bei entsprechend niedrigen Zinsen möglich ist. Wenn die Geldvermögensbesitzer nur wenig am Kauf von Geldvermögenstiteln sowie an der Spekulation mit ihnen verdienen können, werden sie sich stärker zur Anlage in Realvermögen veranlaßt sehen.

Eine produktivitätsorientierte Lohnpolitik, die weder ,zurückhaltend“ noch „expansiv“ ist, stabilisiert das Kostenniveau und verhindert damit Inflation wie Deflation. Lohnzurückhaltung, die hinter dem Produktivitätswachstum zurückbleibt, senkt das Kostenniveau und damit auch - verzögert - das Preisniveau. Im Rahmen einer Währungsunion wie dem Euro-Gebiet würde sie zu einem deflationären Lohnsenkungswettlauf führen, aber nicht automatisch zusätzliche Investitionen anregen. Da Investitionen vor allem von 
den Zukunftserwartungen und dem Grad an Unsicherheit abhängen, wäre ihnen stabilitätsorientierte Lohnpolitik, die Unsicherheit vermindert, am ehesten förderlich.

Allein schon aufgrund ihres Umfangs sind die Staatsausgaben eine gesamtwirtschaftlich bedeutsame Größe. Jede finanzpolitische Entscheidung hat daher notwendigerweise makroökonomische Auswirkungen, die in Rechnung gestellt werden müssen. Wird versucht in wachstumsschwachen Phasen den Haushalt zu konsolidieren und strukturelle Defizite abzubauen, dann wird die Nachfrageschwäche verstärkt. Die Folge ist nicht nur ein weiterer Rückgang der Beschäftigung, sondern auch der Steuereinnahmen, so daß die Konsolidierungsbemühungen konterkariert werden. Daraus folgt, daß rezessionsbedingte Defizite hinzunehmen sind, sie können erst in Wachstumsphasen abgebaut werden (vgl. dazu ausführlicher Priewe 1998a). So war die Konsolidierung des US-amerikanischen Haushalts nur vor dem Hintergrund des starken Wirtschaftswachstum der 90er Jahre möglich. Um Wachstum hierzulande anzukurbeln, wäre eine koordinierte europäische Investitionsoffensive notwendig, die bislang in erster Linie an der konservativen Bundesregierung scheiterte. Die Verschuldungskriterien des Stabilitäts- und Wachstumspaktes, die vom ehemaligen Finanzminister Waigel und der Bundesbank maßgeblich durchgesetzt wurden, blockieren aber die Fiskalpolitik. Wer eine europäische Geld- und Währungspolitik ermöglicht, aber die Geldpolitik fesselt und die Fiskalpolitik auf jahrelange Restriktion programmiert, darf sich weder über schwaches Wachstum in Europa noch über hohe Defizite wundern. Vorschläge, wie etwa investitionsorientierte Verschuldung nicht auf die 3\%-Defizitquote anzurechnen, gehen in die richtige Richtung, werden aber viel zu zaghaft diskutiert.

Um die hohe Zinslast im Bundeshaushalt zu mindern und damit die Schuldenlast der deutschen Vereinigung anders zu schultern, sind Steuererhöhungen, etwa über eine Vermögensabgabe, pauschalen Ausgabenkürzungen in jedem Fall vorzuziehen. Die rotgrünen Austeritätspolitiker sollten auch bedenken, daß die deutsche Staatsausgabenquote, also der Anteil der Ausgaben von Bund, Ländern und Gemeinden am Bruttoinlandsprodukt, bereinigt um die vereinigungsbedingten Zinslasten, im internationalen Vergleich immer noch ziemlich niedrig ist. Inzwischen ist es leichter geworden, öffentliche Armut zu identifizieren als öffentliche Verschwendung, wichtige öffentliche Zukunftsaufgaben werden nicht angepackt.

Vielleicht hätten die Grünen - wie die Sozialdemokraten - noch das Potential, eine alternative Strategie einzuschlagen und zu versuchen, den Teil ihrer Anhängerschaft, der mittlerweile resigniert hat und ins Heer der Nichtwähler oder anderswohin abgewandert ist, zurückzugewinnen. Aber diese Strategie wäre natürlich ebenfalls riskant. Vor allem aber würde sie zweierlei voraussetzen: Erstens, daß sich grüne Politik wieder stärker von eigen- 
ständigen Erkenntnisprozessen leiten läßt, die sich an den Interessen der von sozialen und ökologischen Krisenprozessen betroffenen Menschen orientieren, als von ideologischen Versatzstücken, die ihr von einer politischpublizistischen Elite vorgekaut werden: Globalisierung, Nachhaltigkeit, Generationengerechtigkeit, Bürgergesellschaft, um nur die wichtigsten zu nennen. Und zweitens, daß Grüne wie Sozialdemokraten bereit und in der Lage wären, die zur Durchsetzung eines sozial-ökologischen Reformprojektes notwendigen Staatsaufgaben klar zu definieren und in den dann anstehenden gesellschaftlichen Auseinandersetzungen auch dafür zu streiten.

\section{Literatur}

Bender, St. u.a. (1999): Förderung eines Niedriglohnsektors: Die Diagnose stimmt, die Therapie noch nicht. IAB-Kurzbericht Nr. 6 vom 14.6.1999.

Berninger, Mathias u.a. (1999): Initiative für Investitionen, Arbeit und Umwelt, hrsg. von der Bundestagsfraktion Bündnis 90/Die Grünen, Bonn 16.3.1999.

Bündnis 90/Die Grünen im Bundestag. Arbeitsgruppe Haushalt (1999): Nachhaltige Finanzpolitik: Konsolidierung und wirtschaftliche Stabilität. Diskussionspapier, Bonn, 8.3.1999.

Priewe, Jan (1998): Die Öko-Steuer-Diskussion. Positionen und Kontroversen - eine Bilanz. Berlin 1998.

- (1998a): Staatliche Verschuldungspolitik und die Möglichkeiten expansiver Fiskalpolitik, in: Kommune, Heft 4/1998, S.45ff.

- (1998b): Leitplanken statt Schranken. Wirtschaftswachstum und nachhaltige Entwicklung müssen sich nicht ausschließen, in: Politische Ökologie, Sonderheft „, Wege aus der Wachstumsfalle “, Januar/Februar 1998, S. 30ff.

Reich, Robert (1999): Goodbye, Mr. President: Aus dem Tagebuch eines Clinton-Ministers, München.

Ross, Jan (1999): Ein neuer Glaube, in: Die Zeit, Nr. 29, 15. Juli 1999, S. 3. 